\title{
Beyond the person: the construction and transformation of blood as a resource
}

Rebecca Lynch (LSHTM) and Simon Cohn (LSHTM)

\section{ABSTRACT:}

Many studies of blood donation have looked at the motives of donors, their relationship with the wider society, and corresponding values such as gift-giving, altruism and responsibility. These underpin a rhetorical representation of person-to-person donation that neglects the many technical processes that take place between donation and eventual use and the material nature of blood itself. This ethnographic study, conducted in four UK blood donation sites, describes the various practices involved in routine sessions, rather than the motives or values or donors or staff. It focuses on the procedures and equipment that not only ensure blood is collected safely and efficiently, but the extent to which they determine the nature of the collected blood itself. Taking our cue from posthuman approaches, we argue donated blood as something that is 'made' only when it leaves the body; in other words, it is not simply extracted, but is constructed through specific practices. We illustrate how, as blood is separated from the body, it is increasingly depersonalized and reconstituted in order to have biomedical value. In this way, rather than reproduce the essentialist claim that blood is what social scientists often described as a 'special kind of substance', we point to the ways in which donated blood alters as it moves in time and space. We argue that such transformations occur in both symbolic and material realms, such that the capacity of blood to have both cultural meaning and clinical value is dependent on the fact that it is never stable or singular.

KEY WORDS: blood donation, ethnography, posthuman 


\section{Beyond the person: the construction and transformation of blood as a resource}

The seminal work by Titmuss on blood donation in the 1960s framed UK blood donation as voluntary and altruistic, in which donor and recipient are not known to each other (1987[1970]). His argument is that this both reflects, and reproduces a sense of civic duty and fosters generalised social cohesion and continuity. However, rather than merely provide a sociological commentary, these sentiments have themselves become engrained in the ethos of the nation's blood service. The alignment between public health, blood as a public good and the ethics of civil society continue to be a key idiom adopted by participants in their motivation for donation (Cohn, 2016; Busby, 2004), and utilised by NHSBT (National Health Service Blood and Transplant, the UK national blood service) to promote, recruit, and retain donors, often through their slogan of 'vein-to-vein' donation.

Whilst most people may well be aware that in practice blood is not transferred directly in this way, it remains a compelling representation that does a great deal of work. The motif of human-to-human donation is therefore a useful fiction, reinforced in posters, advertising and webpages. What is intriguing is that this narrative also continues to be reproduced in much of the social science research on the topic, which invariably focuses on the different ways the exchange of blood establishes social ties though its status as a gift (Tran, Charbonneau and Valderrama, 2013; Mahon-Daly, 2012; Wynne Busby, 2010; Healy, 2000). Over thirty years ago, Gregory (1982) noted that gift-based economies comprise of exchanging 'inalienable' goods (objects that are invested with personhood and social relationships). In contrast, in order for objects to be bought and sold they must not embedded in, or restricted by, social ties; hence, commodity-based economies exchange 'alienable' goods (objects without personal attachment). This perspective was taken up by Strathern, in her reflections on the social relations formed through the exchange of various bodily materials (1991). She proposed that while certain human tissues are inalienable, others circulate much like commodities 
because they are largely regarded as entities independent of persons; for example, in contrast to human eggs, she claims human kidneys are not thought about in terms of their social identity. Copeman, however, disagrees (2005), arguing that all human tissue and organs are inalienable to some degree, even if this manifests in different ways. According to him, although such things as donated kidneys or blood might appear to circulate as impersonal objects, the very powerful motives and meanings donors invest in their acts of donation cannot be neglected, even if they are not always carried overtly along with the donated material $(2005 ; 479)$.

Between these opposing stances, Waldby suggests that human tissues such as donated stem cells and blood are never either commodities or gifts (2002), pointing out not only that these different spheres of exchange usually co-exist, but that there is rarely a clear demarcation between them. She suggests that what should be attended to instead is the circulation of new forms of 'biovalue' which are rapidly emerging from the 'biotechnical reformulation of living processes.' Nevertheless, in common with all these positions, the issues of anonymity and personhood are presented as key to determining the alienability or otherwise of substances such as blood, and therefore the manner in which it might circulate. And in keeping with Gregory's original concern, it is assumed that the determination of this is a cultural process, irrespective of its material nature.

In contrast to this, our argument is that matters of alienability are not solely determined by a substance's symbolic value, and that producing blood for future clinical use or research requires a combination of material as well as semiotic transformations. If one fails to consider the extent to which blood materially alters as it is collected and redistributed, the same fiction that donors and the blood service draw on is inadvertently reproduced. Berner (2010) provides a fascinating historical account of developments of blood transfusion over the first half of the twentieth century, during which time the increasing number of medical techniques 
and interventions embedded the donor and recipient in more and more complex sociotechnical assemblages. The result was that the donor became increasingly separated from the recipient, both physically and temporally. Our paper complements this insight, but focuses on the contemporary ways in which routine procedures not only separate donors' blood from their body, but how this separation in inescapably coupled with its material alteration. In this way, it problematizes any description that final blood products simply come from a donor's body.

This approach draws on shifts over recent years from a diverse range of perspectives to reframe conventional social sciences approaches by not assuming the human is necessarily the principle agent in social and cultural events (e.g. Braidotti, 2015; Wolfe, 2010). Such approaches have included thinking about the role of non-human organisms, and the relationships they have with humans (Haraway, 2008); the significance of material objects and the extent to which their physical properties shape how events unfold (Barad, 2007); and more generally, how any specific context is not merely the background for a discrete set of agents, but is a heterogeneous field which actively determines the formation and configuration of agents themselves (Puig de la Bellacasa, 2011). Arguably one of the most famous essays which set out much of this intellectual agenda was by Callon in his discussion of French fisherman, scallops, and attempts to farm them (Callon, 1984). In this account, he describes the ways in which different elements - humans, objects, physical environments and non-human organisms - relate and associate with each other in such intricate ways that often human agency and even political potential is dependent on the manner in which many nonhuman elements may 'choose' to act. A key point he raises is that in the making of relationships, the 'identity and characteristics of the implicated actors change as well'. 
Overall, this broad literature, which has collectively been described as 'posthuman', does not dismiss human accounts, but rather recognises the productive value of resisting traditional assumptions about what is significant, valuable, or relevant. Our view, therefore, is that the term itself can often be unhelpful, in that it suggests a primary interest in people is unequivocally displaced by these new orientations: This is not necessarily the case. What is more crucial is that such approaches remain vigilant to the many nonhuman elements - for example, the physical environment and role of material objects - since they do not simply enable human action, but form an integral part of what that action is and can do. Following Latour (2005), this produces a radically different notion of what should constitute social enquiry because it emphasises the heterogeneity of potential actors and members of the social, inviting a fuller, and indeed more inclusive, ethnography (Panelli, 2010). As Mol has pointed out, social researchers often forget about the many other actors in the room (Mol, 2002). By broadening the scope of enquiry not only to include non-human elements but recognise them as potential actors, humans may be said to be decentred. But this does not mean that our primary matter of concern, rather than scope of enquiry, can no longer continue to be that relating to humans and their health.

The value of adopting this perspective to think about blood donation is not only that we might resist falling into the trap of thinking of donation in terms of the simple exchange from one person to another. It might also engage with the significant modernization of services in recent years within the UK and internationally, that has led to the introduction of new equipment, new practices, and ultimately new forms of biovalue (Alliance of Blood Operators, n.d.; Australian Red Cross Blood Service, 2014). Changes to biomedical science and research is shifting the relations between the commercial sector, public healthcare and the individual; as a result, discourses of waste and efficiency are increasingly being combined with those of gift-giving and altruism (Tupasala, 2011). Developments are also influencing the demand for 
blood, and in particular the traffic of specific blood components and products in a global 'technoscape' (Appadurai, 1996). At a national level, while the UK blood service is typically associated with small mobile donation sites in local communities it is progressively placing greater emphasis on electronical appointment sessions run from custom-built static centres, which are better able to respond to fluctuations in demand for blood and blood products. Coupled to this, increased attention is being paid to the 'safe donation experience', promoting a 'modern, attractive and easy to use service', ensuring 'high levels of customer service' and 'reducing the variation in experience for donors' (NHSBT a, n.d.). NHSBT working papers use phrases such as developing a 'lean national supply chain' and 'minimising wastage, reduce infrastructure costs and minimise non-productive time' (NHSBT b, n.d.). Accordingly, new protocols, equipment and procedures are being introduced to ensure donation sessions are as efficient as possible and increasingly able to respond to contemporary demands.

However, rather than address these developments through an argument of globalising 'neoliberal' processes of efficiency and homogenisation (Will, 2017; Bell and Green, 2016), we instead examine the effects that these forces shape local practice, and the way relatively small technical procedures serve to enact them. Against the backdrop of a complex and distributed international system of blood exchange, there may well be increasing dissonance with the traditional vein-to-vein, human-to-human, motif, demanding new ways to think about blood, its movement, and its value. As a consequence, we are interested in the ways that these changes may relate to, or clash with, the continued promotion of social and cultural meanings attached to blood and blood donation. This paper therefore asks to what extent might the growing imperative to increase the biovalue of blood in the national and international market, as articulated by ideas of efficiency and leanness, inevitably strip the social and cultural values from donated blood. 


\section{Methodology}

In this paper, we draw on ethnographic observations of donation sessions and interviews with donors. This approach allows examination not only of what people say and do, but also the active role materials, objects and technologies have. Donation sessions in four of NHSBT's current 24 static centres established were observed over eight months; one based in a community hospital, two in large hospital sites (one urban, one more rural) and the fourth within an urban shopping centre. The purpose was not to compare these sites, but rather identify those aspects of procedure, equipment and practice that they had in common. Our approach was inspired by the much-cited advice from Actor Network Theory to 'follow the actor" (Latour, 2005) - in our case, the flow of blood. However, knowing that there were many different, if apparently minor, procedures and practices involved in determining who might donate and what blood was acceptable, our approach was not simply to record what networks blood became enmeshed in, but rather how those interactions served to constitute blood in particular ways. In other words, by taking blood to be our ethnographic focus, rather than the people, our analysis attended to changes in matter, and well as meaning.

We also observed various NHSBT meetings, chatted informally to staff (donor carers, nurses, centre managers and receptionists) and conducted interviews with thirty regular blood donors about their experiences. The donors were drawn from participants in a randomised control trial looking at the impact of increased frequency of donations on blood supply and donor health (the INTERVAL trial; for more information see Moore et al, 2014) and were purposefully sampled to reflect a range of ages and balance of gender. The in-depth semistructured interviews were conducted by RL, lasted between 45-145 minutes and were audiorecorded prior to thematic coding and analysis. Verbatim transcriptions were imported into NVivo 10 alongside the field notes. All data was initially coded using themes from the interview guide and then using more analytical codes which emerged through systematically 
reading and re-reading the transcripts. $\mathrm{RL}$ and $\mathrm{SC}$ discussed and refined these iteratively in order to establish higher-level themes. Throughout the study we were sensitive to the needs and positioning of donors and donation centre staff, ensuring that we did not impede their activities, and only directly observed or interviewed individuals after explicit consent to do so. Formal NHS ethical approval was obtained for this study.

Below, we bring together the observations, reflections from the interviews, and descriptions of some of the techniques, protocols and materials involved in contemporary blood donation to give an account that centres on the non-human elements of donation, and the ways in which these interact with human actors to produce certain material entities. In so doing, we detail how blood undergoes a series of necessary alterations in order that it will be suitable for recipients at a later date. The process begins with separating bodies from persons, then blood from bodies, and finally a separation of blood itself. These processes of separation and division are also accompanied by various additions and modifications. We consequently highlight how blood is never a singular or essential substance, but is always a composite that can always be further divided or augmented to produce new biomedical potential.

\section{Separating bodies from people}

Individual donation sessions follow a national standardised procedure developed by NHSBT that stipulates not only the order of various steps, but many other details, such as the arrangement of equipment and layout of furniture. Instructions are designed to make the process as seamless and orchestrated as possible. In doing so, the clear linear ordering not only plots out the various stages and techniques, but communicates the overall message that successful sessions are about managing throughput and the efficient attainment of blood. 
Potential donors must be aged between 17 and 66 if it's their first time, although currently there is no upper age limit for existing donors. Before any physical tests are conducted, they must all complete a preliminary form called the 'Donor Health Check'. This requires them to tick boxes about their lifestyle, sexual behaviour, current health status, medication, and periods of travel outside the UK. Everyone is aware that the questionnaire is designed to screen out people who might have a high risk of blood-borne infection: those who have had recent piercings or tattoos; who have travelled to particular countries where the likelihood of contracting certain illnesses are higher; those who have injected drugs; or might be at risk of carrying HIV because of their recent sexual activity. In this way, completing the form already starts to select those who can donate from those who cannot. But throughout much of the promotional work it does, the blood service is very careful about navigating between, on the one hand, being able to reassure people it is continually vigilant about the safety of its procedures and products, and on the other animating people's sense of good will and altruistic intentions. It is an almost impossible tension; some people object to filling in the questionnaire or to certain specific questions, while others may see the need in general, but take offense that somehow they themselves are not being trusted.

A brief private follow-up interview by a qualified nurse goes over the responses to confirm suitability. Those deemed to be 'low risk' then have a droplet of blood taken to test whether they have sufficient levels of haemoglobin. A finger is pricked and a blood droplet dripped into a blue-coloured aqueous copper sulphate solution. Its specific gravity means that only blood containing a sufficient amount iron is able to descend. If the blood fails to sink, a follow-up test is done using blood from a vein and an electronic meter. Anyone confirmed to have low levels of iron, and who might potentially be diagnosed anaemic, is told to come back after some weeks. Again, NHSBT is very conscious of the potential effects of deferring people in this way, and the fact that some people may experience this as rejection so never return. So when 
this does occur, nurses generally follow a scripted response to encourage the potential donor to come back, reflecting the fact that retaining an active donor population is a constant problem, as newly recruited donors tend to be continually offset by those who no longer turn up.

Through these initial routine stages of selection there is a clear progression from engaging with the donor as a person, by asking questions about their lifestyle and behaviour, to increasingly concentrating on the body and the nature of the blood it contains. In this way, persons are progressively separated from their bodies. The various arrangements and items of kit are key to a process designed to protect the safety of donors and guard against any possible contamination of the blood; from the questionnaire, to the iron test, the focus of attention shifts from care about the lifestyle and experiences of the donors to care of the body as a container of blood. The staff, too, are part of this selection technology. However, they find the task of selecting donors from those who have made the effort to come, but have to be turned away, tricky. Matters relating to the cultural and the biological have to be divided, as the symbolic significance of blood drawn on to encourage people to come potentially conflicts with having to assess bodies and blood according to different criteria.

Those judged as being able to donate have to wait until a slot becomes available. They are then greeted by a donor carer, and walked over to a specially-designed chair. The chairs are increasingly being used at centres because they have been specifically designed to provide a safer and more ergonomic alternative to the traditional beds, which offered limited adjustment and couldn't always prevent donors from falling off. The chair can be swung rearward to help prevent fainting, or reclined fully to allow recovery should this occur (fainting occurs in around 300 blood donors in a typical donation day, SEE Platform, 2014). Once seated, the carer checks the details on donor's form, and makes sure that everything matches on the multiple sets of labels that will be used. A sealed collection set is then opened. Described as a 'Whole Blood Collection and Component Storage System', it is made up of a main blood bag 
(which contains an anticoagulant and an additive to ensure the blood remains viable), a sample pouch, tubing and a catheter needle. The contents are carefully inspected, checked to see it is in date, and that there is no sign of moisture (which may indicate a leak).

Then, during the actual period of taking blood, donors freely allow their allotted carers to take control, touching and moving their bodies to determine how things should proceed. The carer normally asks which arm the donor would like the blood to be taken from. A conversation about veins might then ensue while a tourniquet is put on, pulled taut, and a suitable vein is felt for. Frequently the donor and staff member inspect the arm jointly; in so doing, the limb is presented between them as a common, but distinct, object. The language used also reflects this somewhat detached assessment, with regular comments about which veins might be 'good' or 'problematic' depending on whether they are thought able to hold the standardsized needle and facilitate a good flow. Some donors even boast about the speed at which their arm or blood vessels can usually fill a blood bag (usually between 5-10 minutes). In this way, through the joint interaction between the donor and staff, it is now the physical properties of the person's body which are now being scrutinised. While the cuff maintains pressure, and the puncture site is checked for skin lesions and made sterile, a 16-gauge cannula needle is taken out of its tamper-proof cover. At this point, although some donors cast their gaze away, but many watch with fascination as it enters the arm and blood is drawn. First, a small amount flows through the collection tubing into a sample pouch. Being physically part of the collection kit, this in-line system reduces overall donation time and any risk of bacterial contamination. But once a sample has been collected, a permanent clamp is clicked shut (which will allow for the pouch to be snapped off later) and the flow diverted to the main collection bag.

While a few may continue to avert their eyes as the blood flows, most are clearly intrigued, looking with interest as their blood streams down the tube into the blood bag. Some donors 
spoke afterwards about the uncanny feeling of blood flowing from their arm, and that its warmth though the tubing felt particularly strange against their cooler skin. As the blood is collected donors may watch televisions positioned above them, read, or use the internet on their phones. To ensure the blood, anticoagulant and additive are gently mixed together, the blood bag is retained in a rocking agitator that inverts it every 60 seconds. It makes a gentle repetitive mechanical noise. Staff who had worked in the service for a long time recalled how, before these devices were introduced, the mixing of blood and anticoagulant had to be done by hand continuously until the bag was full. Regulating this is now an automated process, as the agitator also constantly weighs the blood and then stops, emitting warning beeps, when 470-475 $\mathrm{ml}$ has been collected.

Despite this degree of standardisation, individual carer staff often have their own preferred ways of using the equipment; for example, differing slightly in the ways in which they may place the tape on an arm to hold the needle or 'tricks' to improve the standard venepuncture protocol by arranging the tubing in particular locations to aid flow. The brief chats with the donor, constant attention to the arm, and regular checking of the equipment, are ways in which staff move between caring for the donor, caring for the body, and caring for the blood as it is produced. It is their role to manage these distinct remits: to produce a full bag of blood filled to correct levels and mixed with coagulant; a body which produced a timely and continuous flow of blood without incident; and a person who doesn't feel unwell or faint.

Because identical equipment has to be used - the same needle for big or small veins, the same chairs for tall or short bodies, the same volume blood bag - their design mean that occasionally staff are unsuccessful in collecting a full bag of blood. Sometimes a suitable vein cannot be found, or the flow is too sluggish, or occasionally the donor feels such discomfort that the procedure has to be halted. This means that, in practice, staff consider donation to 
be generally easier from male donors (who typically have larger veins) and people who are not too small. When asked about this, one donor carer simply responded by saying 'you can't change the equipment, you have to change the body', referring to the fact that on rare occasions if they fail in collecting blood from someone they simply move on to the next person waiting. In this way, the design of the technology does not simply reflect norms but also operationalises them, itself sorting out variations of persons and bodies into those that are able to donate from those which cannot. In so doing, different elements interact - the chair, the arm, a cannula, a vein - each of which can contribute to determining not only how the blood will be collected, but the very nature of the collected blood itself.

Once the rocking agitator machine indicates that the bag is full, the carer deflates the pressure cuff, removes the catheter, tubing and blood bag from the arm, and asks the donors to hold a cotton swab over their puncture site. The needle is placed in a specialised disposal container and the blood bag is gently inverted a few more times by hand to make sure its contents has been thoroughly mixed. Donors are told briefly about how to look after their bodies; they are advised to keep pressure on the bandage, drink plenty of fluids, rest and not exert themselves, not drive immediately, and not have a hot bath that day. They are warned to look out for feeling light-headed or dizzy, in which case they should lie down immediately. And after this, they are directed towards a seating area to have a cup of tea or fruit drink and a biscuit to recover: time, liquid and sugar all designed to avoid the possibility of fainting.

While the donor rests, the carer takes the blood bag and sample pouch to the packaging area. Here it is categorized by type and Rhesus status, and the data added to charts and tallies in order to see how the session compares to the weekly targets set for different blood types. Already by this stage of the donation procedure, the blood has undergone processes and operations which not only separate it from the donor, but have changed its material nature and qualities. It is mixed with new substances and contained in different vessels that alter its 
physical characteristics; for example, as it cools it no longer coagulates because of new chemical bonds that have been introduced, and the cells do not die rapidly. And in this form it now has new biomedical potentials.

\section{Separating blood itself}

When talking about the donation process afterwards, donors convey little sense of ownership of their blood once it has left their arm, with only a few reflecting on whether they even saw a van or bike transporting blood away. The blood is packed into cool bags and picked up by specially trained couriers and taken to one of the large national processing centres, while the sample pouch is sent separately for testing at sites which provide high throughput laboratory analysers for signs of HIV, hepatitis B and C, Human T-cell Lymphotropic Virus (HTLV), and syphilis. The advanced IT system, called 'Pulse', ensures barcode linkage between this and the trajectory of the main donation - what is frequently termed 'traceability'. Although not a secret, NHSBT rarely talks about processing centres in its promotional materials. They are large, industrial-like buildings, housing staff and machinery, including long conveyor belts, crates of blood bags, and robotic handling in large open spaces.

A key element of what NHSBT call the 'blood supply chain' is the main PVC bag itself. It has been designed not only for collecting the blood but also for transit and processing, thereby maintaining a 'closed system' to minimise waste or the possibility of contamination. The flexible bags are strong enough to ensure they do not accidentally tear or burst, yet are also permeable because platelets need oxygen to survive, and are heat resistant so they can sterilised using steam (a cheaper method than radiation sterilisation). This relatively simple technology has had a great effect on NHSBT operations, allowing for a high degree of automation throughout various stages, and making direct human intervention redundant. It enables blood processing to be conducted 24 hours a day, seven days a week using advanced systems in blood filtration, conveyor and storage system. 
At the processing centre, the bagged blood is sorted, registered, and then sent to what NHSBT terms 'the manufacturing area'. Small amounts of rare blood types may be frozen whole, but most blood is separated into different components. Having filtered out white cells, the bag is centrifuged or 'pressed' to divide - or 'fractionate' - red blood cells, plasma and platelets into different packs that are sealed and detached from each other. These different substances are then held in 'quarantine' until the results of the blood test confirms whether they can be used. After this, the packs are placed in storage, where an even temperature distribution is maintained and vibrations, which might damage the blood components, are minimised. The requirements of each varies, further illustrating the extent to which they are now discrete entities. Red blood cells can be kept for 42 days at a temperature of between $2^{\circ} \mathrm{C}-6^{\circ} \mathrm{C}$; the platelets only last up to seven days and are stored in incubators at room temperature and are agitated once a second to prevent clumping; and finally the yellowish plasma is frozen at below $-18^{\circ} \mathrm{C}$, known as cryoprecipitate, where it remains viable for a year.

This description shows how the blood produced at local centres is now combined and divided in new ways as it is filtered, separated, and tested, to be radically transformed. NHSBT describe these technological manipulations as 'manufacture', creating substances that are more and more distinct from the blood that was once circulating in the donor. But it is only through these manipulations that the products have the potential to circulate in different bodies. Each will go on to be used for different purposes; the red blood cells are mostly used for trauma and surgery, but also for treating anaemia, blood loss and for blood disorders such as sickle cell anaemia; platelets are generally used for cancer treatments and organ transplants; while the plasma is commonly used to treat liver and kidney disease and bleeding disorders such as haemophilia. These products have not simply been 'extracted' from the blood; while they were in the blood bags they existed in combined form as donated blood. 
Now, they exist in their new forms through processes involving humans and nonhuman to enable them to materialise, be stored, and transported in new ways.

\section{Discussion}

At the beginning of this paper, we outlined how overly human-centred accounts of blood donation give the impression that blood is inevitably a special substance and is afforded with an exceptional status because of its intrinsic cultural and symbolic positioning. NHSBT often reproduces this motif when promoting the service and seeking new donors by focusing on how a person giving blood can 'save a life' and establish a direct person-to-person tie with an anonymous recipient ${ }^{1}$. Our paper has been an attempt to address two aspects of that representation which has led to much research focusing solely on the cultural symbolism of blood donation and its social meaning. The first, is that this concentrates on human actors at the expense of many other elements that are just as significant for blood to be successfully collected, distributed and used. And the second is that it depicts a continuity of the material nature of blood throughout the stages of exchange. In contrast, by attending to the mundane, material aspects of blood donation our ethnography describes some of those aspects frequently left out. We have drawn on a posthumanism sensibility to de-centre the role of the donor and highlight particular elements and relationships not normally included. In particular, we have described not only the various stages of material transformation, but the ways in which these have to be accomplished without completely undermining the social and cultural representations of blood donation.

As a feature of these processes, we illustrated how particular entities are divided and excluded - types of persons, types of bodies and types of blood - and how other entities come together - the vein and the cannula, blood and coagulant, white blood cells and a filter, etc. Through these interactions, new forms of blood are produced. The processes alter its composition, 
location, temperature, and potential capacities. The point is that in accounts that describe the movement of blood from one location to another, and from one time point to another, it cannot be one, stable, substance; like all entities, as it travels it inevitably alters (see Williams, 2013). The stages of production, distribution and consumption, drawn on in many accounts of commodities, including pharmaceuticals and biological products, tend to present them in a simple linear fashion, leading to a focus on such things as medicalisation, pharmaceuticalisation, regulation and consumption (see for example, Williams, Gabe and Davis, 2008). But thinking about donation as the first of these would keep particular actors and bodies separate, presenting blood as a stable substance that passes along from one to the next. In our study, many aspects of transportation, distribution and the future use of the blood determined by national and global forces shape the practices of donation itself.

The result is that blood is continuously manipulated as a range of actions alter and transform its capacities and potentials; it is stored and tested, has products added and components removed, is frozen, separated and reassembled, and is transported from arm to bag, and from local donation sites to national processing centres. Although blood may well continue to be a powerful signifier for such things as kinship, race and the social collective, and ideas of stability and continuity, its biovalue is entirely dependent on a continual chain of transformations. It is precisely this status of blood as a separated and technological set of products that means it can be used by others. The stripping away of some of its biological entanglements with the donor - through processes of selection, filtration, separation and mixing - enables it to then amalgamate with different human bodies at a later date. One reading of this might be to suggest that the transformations of blood produce substances that are themselves 'post' human and less 'natural' than the blood circulating in a donor's body. However, whilst these may initially appear to be processes that make the blood more 'pure' and uniform, less 
'individual' and entirely alienable, we want to argue that instead what is happening is that different human dimensions are being introduced as others are foreclosed.

Although the practices of blood donation may well disentangle aspects of the individual donor from the blood, this is not a simple process of purification to produce blood in some kind of unadulterated state. The clinic and its documents, the specially designed chairs, the courier van with trained driver, the processing centre with its conveyor belts and centrifuges are all enfolded in the bloods they enact because they determine the nature of the substances that advance, and those that do not. It is not simply that the design of the equipment, the protocols of the donor carers, and even the automated systems of blood product manufacture are designed by humans, but that they fashion the materialisation of blood in particular ways. In this way, the transformed blood is no less human - it is just less of one particular human. This point shows how materialist accounts therefore go hand-in-hand with more human-centric accounts, rather than conflict with them. The very existence of blood in separated forms, in bags, kept at specific temperatures, and mixed with new substances, relies entirely on a wide range of technical human operations. Consequently it makes little sense to oppose what is human from what is nonhuman, what is inalienable from what is alienable, since, paradoxically, one term does not simply define the other, but enables it. We therefore are not suggesting that the symbolic aspects of blood and blood donation - relating to such things as life, vitality, vigour and rescue - are simply mistaken because donated blood is not the substance it is assumed to be. Rather, our study points to the fact that essentialist accounts of blood are themselves predicated on the fluidity and multiplicity of the substance itself. In other words, cultural ideas of blood as being tied to personhood, and indeed more general accounts of being human as a singular and stable entity at all, can only be maintained in everyday life by virtue of the fact that material forms constantly reconfigure, adapt and alter. 
At the beginning, we asked whether changes to the blood service, which increasingly are adopting the language of efficiency, production, and manufacture, might clash with existing cultural ideas associated with the donation of blood and saving the life of another person. Certainly, our account touched on occasions when either individual staff members or the organisation itself struggles to divide these two logics. But many of the biotechnical material manipulations and interventions that enable blood to be associated with ideas of social stability and continuity are already witnessed by donors and part of the imagery that NHSBT draw on. This suggests people do not experience these different spheres of meaning - the efficient manufacture of blood products and giving the gift of life - as necessarily contradictory. Rather, it may well be that values of human health are already seen to rely on other kinds of value, that intersect and enable what it means to be human, and what it means to be healthy. This closing observation is an important one for public health. To think beyond the human, by paying particular attention to other non-human actors, does not necessarily mean that public health is no longer primarily concerned with the health of people. But it does point to the fact that how we might value human health, and act to improve it, is always dependent on things we define as not being human. And, as we have shown, people are always embedded in the social, but the world of the social is not solely human.

\section{Acknowledgements}

The authors would like to thank all donors who kindly gave up their time to be interviewed as well as the managers and staff of the blood donation centres for their assistance and hospitality. A version of this paper was presented as part of a panel on post-human approaches in anthropology at the EASA/RAI medical anthropology conference in September 2015 and we are grateful for the comments of participants on this paper. In addition, the authors are grateful for the assistance and guidance of those involved in running the INTERVAL 
blood donation study, particularly Professor John Danesh, Carmel Moore, Zoe Tolkien and Rachel Henry at the University of Cambridge, and Gayle Miflin at NHSBT. Participants in the INTERVAL trial were recruited with the active collaboration of NHS Blood and Transplant (NHSBT) England (www.nhsbt.nhs.uk). Investigators at the University of Oxford have been supported by the Research and Development Programme of NHSBT, the NHSBT Howard Ostin Trust Fund, and NIHR Oxford Biomedical Research Centre.

\section{Funding}

This work was funded as part of the INTERVAL blood donation trial. INTERVAL was supported by core funding from: NIHR Blood and Transplant Research Unit in Donor Health and Genomics, UK Medical Research Council (G0800270), British Heart Foundation (SP/09/002), and NIHR Cambridge Biomedical Research Centre.

\section{Ethics}

Ethical approval was granted by the National Research Ethics Service Committee East of England- Cambridge East (Research Ethics Committee (REC) reference 11/EE/0538. The authors have no conflict of interests. 


\section{References}

Alliance of Blood Operators (n.d.) 2014-2019 strategic plan. Accessed 23/8/16 through: https://allianceofbloodoperators.org/media/101721/ABO-Strategic-Plan-2014-FINAL.pdf

Appadurai, A. (1996) Modernity at large: Cultural dimensions of globalization. Minneapolis: University of Minnesota Press

Australian Red Cross Blood Service (2014). At the leading edge: strategic plan 2014-2019. Accessed 23/8/16 through:

http://www.donateblood.com.au/sites/default/files/Strategic Plan 2014-

2019 At the Leading Edge.pdf

Barad, K. (2007) Meeting the Universe Halfway: Quantum Physics and the Entanglement of Matter and Meaning. Durham: Duke University Press.

Bell, K. Green, J. (2016) On the perils of invoking neoliberalism in public health critique. Critical Public Health 26 (3), 239-243

Berner, B. (2010). (Dis) connecting bodies: Blood donation and technical change, Sweden 1915-1950. In Technology and Medical Practice: Blood, Guts and Machines. Eds. E. Johnson, and B. Berner. Ashgate Publishing, Ltd.

Braidotti, R. (2015) The Posthuman. Cambridge: Polity Press

Busby, H. (2004) Blood donation from genetic research: What can we learn from donors' narratives? In R. Tutton \& O. Corrigan (eds), Genetic databases: socio-ethical issues in the collection and use of DNA. London: Routledge, pp39-56

Callon, M. (1984). Some elements of a sociology of translation: domestication of the scallops and the fishermen of St Brieuc Bay. The Sociological Review, 32(S1), 196-233.

Cohn, S. (2016). Blood and the public body: a study of UK blood donation and research participation. Critical Public Health, 26(1), 24-35.

Copeman, J. (2005) Veinglory: exploring processes of blood transfer between persons. Journal of the Royal Anthropological Institute. 11, 465-485

Gregory, C.A. (1982) Gifts and commodities. London: Academic Press

Haraway D (2008) When Species Meet. Minneapolis: University of Minnesota Press

Healy, K. (2000) Embedded altruism: blood collection regimes and the European Union's donor population. American Journal of Sociology 105:6, 1633-1657

Latour, B. (2005) Reassembling the social: an introduction to Actor-Network-Theory. Oxford: Oxford University Press

Mahon-Daly, P.M. (2012) Blood, society and the gift (A thesis submitted for the degree of Doctor of Philosophy by Patricia Mary Mahon-Daly). Brunel University, UK 
Mol, A. (2002). The body multiple: ontology in medical practice. Durham/London: Duke University Press

Moore C, Sambrook J, Walker M, Tolkien Z, Kaptoge S, Allen D, et al. (2014) The INTERVAL trial to determine whether intervals between blood donations can be safely and acceptably decreased to optimise blood supply: study protocol for a randomised controlled trial. Trials. 15,363

NHSBT a (n.d.), Blood 2020. A strategy for the blood supply in England and North Wales. Accessed 23/03/16 through: http://www.nhsbt.nhs.uk/download/blood-2020.pdf

NHSBT b (n.d.), Strategic Plan 2013-18. Accessed 23/03/16 through http://www.nhsbt.nhs.uk/download/nhsbt-strategic-plan-201318.pdf

Panelli, R. (2010). More-than-human social geographies: Posthuman and other possibilities. Progress in Human Geography, 34(1), 79-87.

Puig de la Bellacasa, M. (2011) Matters of care in technoscience: assembling neglecting things. Social Studies of Science 41(1), 85-106

SEE Platform [Sharing Experience Europe] (2014). Case study on blood donation. Accessed 10/6/16 through: http://www.seeplatform.eu/casestudies/Blood\%20Donation\%20Chair.

Strathern, M. (1991) Partners and consumers: making relations visible. New Literary History. 22(3), 581-601

Strathern, M. (2009) Afterword (to special issue on blood). Body and Society 15(2), 1-28

Titmuss, R. (1987) The gift relationship: from human blood to social policy. London: George, Allen and Unwin

Tran, N.Y.L., Charbonneau, J.,Valderrama-Benitez, V. (2013) Blood donation practices, motivations and beliefs in Montreal's Black communities: the modern gift under a new light, Ethnicity \& Health, 18:6, 508-529

Tupasala, A. (2011) From gift to waste: changing policies in biobanking practices. Science and Public Policy. 38(7), 510-520

Waldby, C. (2002) Stem cells, tissue cultures and the production of biovalue. Health: An Interdisciplinary Journal for the Social Study of Health, Illness and Medicine. 6(3), 305-323

Will C.M. (2017) On difference and doubt as tools for critical engagement with public health. Critical Public Health, 27(3)

Williams, J. (2013). Gilles Deleuze's Difference and Repetition: A Critical Introduction and Guide. Edinburgh University Press.

Williams, S. J., Gabe, J., Davis. P. (2008) The sociology of pharmaceuticals: progress and prospects. Sociology of Health \& IIIness 30(6), 813-824. 
Wolfe C (2010) What is Posthumanism? Minneapolis: University of Minnesota Press

Wynne Busby, H. (2010) Trust, nostalgia and narrative accounts of blood banking in England in the $21^{\text {st }}$ century. Health 14,369

\section{Notes}

1 In Sweden, this has been reinforced through a campaign which sends donors a text when their blood has been used for another person. Here the processing and splitting that donated blood undergoes is not flagged up but rather the direct link between donor and recipient is maintained 